\title{
Agreement between a physiotherapist and an orthopaedic surgeon regarding management and prescription of corticosteroid injection for patients with shoulder pain
}

Darryn Marks ${ }^{a, b}$, Tracy Comans ${ }^{b, c}$, Michael Thomas ${ }^{a}$, Shu Kay Ng ${ }^{b}$, Shaun O'Leary ${ }^{d, e}$, Philip G Conaghan ${ }^{f}$, Paul A Scuffham ${ }^{b}$, Leanne Bisset ${ }^{b}$

\section{Author affiliations}

a Gold Cost Hospital and Health Service, Gold Coast University Hospital, 1 Hospital Boulevard, Southport 4215, Gold Coast, Australia.

${ }^{\mathrm{b}}$ Menzies Health Institute Queensland, Griffith University, Parklands Drive Southport, QLD 4222 Gold Coast, Australia.

c Metro North Hospital and Health Service, 112 Alfred Street, Fortitude Valley QLD 4006, Brisbane, Australia.

${ }^{\mathrm{d}}$ School of Health and Rehabilitation Sciences, University of Queensland, Brisbane St Lucia, QLD 4072, Australia.

e Physiotherapy Department, Royal Brisbane and Women's Hospital, Butterfield Street, Herston QLD 4006, Australia.

${ }^{f}$ Leeds Institute of Rheumatic \& Musculoskeletal Medicine, University of Leeds, \& NIHR Leeds Musculoskeletal Biomedical Research Unit, Chapel Allerton Hospital, Leeds, LS7 4SA, UK.

\section{Correspondence:}

Darryn Marks

Allied Health, Gold Cost Hospital and Health Service, Gold Coast University Hospital, 1 Hospital Boulevard, Southport 4215, Gold Coast, Australia.

Email:darryn.marks@health.qld.gov.au

Ph: +61756873120 


\section{ABSTRACT}

\section{Background:}

Physiotherapists increasingly manage shoulder referrals in place of orthopaedic doctors. Better understanding the agreement between these professionals will help inform the safety, quality and potential costs of these care models.

\section{Objective:}

To establish the level of agreement between a physiotherapist and an orthopaedic surgeon regarding diagnosis, management and corticosteroid injection, in a representative sample of orthopaedic shoulder referrals.

\section{Design:}

Blinded inter-rater agreement study.

\section{Method:}

274 public orthopaedic shoulder patients were independently assessed by a physiotherapist and an orthopaedic surgeon. Management, subacromial corticosteroid injection, diagnosis and investigation decisions were compared using inter-rater reliability statistics.

\section{Results:}

Agreement between the physiotherapist and the orthopaedic surgeon was near perfect for surgical versus nonsurgical management (Gwets agreement coefficient 
$\mathrm{AC1}=.93,95 \% \mathrm{Cl}: .90-.93)$, safety of injection (AC1=.85, $\mathrm{Cl}: .79-.91)$ and investigations requested $(\mathrm{AC1}=.87, \mathrm{Cl}: .83-.91)$; substantial for the presence of subacromial pain (AC1=.74, $\mathrm{Cl}: .66-.81)$ and diagnosis (AC1=.72, $\mathrm{Cl}$ : .66-.78); and moderate regarding delivery of subacromial corticosteroid injection as an immediate treatment $(\mathrm{AC1}=.48, \mathrm{Cl} .33-.53)$, with the physiotherapist less inclined to select corticosteroid injection as the first intervention.

\section{Conclusion:}

In this study a physiotherapist with prescribing and injection training made decisions analogous to those of an orthopaedic surgeon at initial consultation for orthopaedic shoulder pain, including the safe identification of patients for subacromial injection, without the need for prior screening of referrals by orthopaedic doctors.

Trial registration: Australia and New Zealand Clinical Trials Registry, number XXXX

\section{Keywords}

shoulder, corticosteroid injection, physiotherapy, orthopaedic 


\section{BACKGROUND}

Musculoskeletal disorders are the second largest cause of global disability (Vos et al. 2012), with prevalence expected to increase as the population ages (Murray et al. 2012). As publicly funded health services face growing challenges (Australian Government Productivity Commission 2011; Department of Health 2006; The Kings Fund 2015), orthopaedic screening services increasingly provide early access to experienced physiotherapists (Comans et al. 2014; Desmeules et al. 2012; Stanhope et al. 2012) for assessment and non-surgical management. As the second to third most prevalent musculoskeletal disorder (Parsons et al. 2007; Picavet and Schouten 2003; Taylor 2005; Urwin et al. 1998), shoulder pain is frequently referred for orthopaedic opinion (Buchbinder et al. 2013; Dorrestijn et al. 2011; Linsell et al. 2006) and often seen by physiotherapists in screening services (Queensland Health 2013) where decisions made by physiotherapists, rather than medical doctors, determine patient care. Consequently, information is needed about the level of similarity between physiotherapist and orthopaedic surgeon decisions, to better understand the safety, service quality and potential costs of emerging models of care in which patients are seen by physiotherapists instead of medical doctors.

Prior comparisons of physiotherapist and orthopaedic surgeon decision-making only partially represent emerging models of care, as they focused on less complex 
patients and neglected prescribing and corticosteroid injection. Physiotherapists and orthopaedic surgeons generally agree on hip and knee diagnosis and management (Aiken et al. 2008; Aiken and McColl 2008; Desmeules et al. 2013; MacKay et al. 2009), however all but one study (Aiken et al. 2008) excluded patients with higher comorbidity or complexity. Only two studies have investigated shoulder pain; one had only six shoulder participants (Aiken and McColl 2008), the other reported a Kappa of 0.75 for surgical versus conservative management (Razmjou et al. 2013) and both excluded more complex cases with suggestion of infection, metabolic or inflammatory disease, masses, compensation claims or previous surgery. Therefore, no studies have yet compared the shoulder management decisions of a physiotherapist with those of an orthopaedic surgeon, across the range of presentations that might normally attend an orthopaedic outpatient clinic. This knowledge gap has important application to service design, regarding whether it is necessary for an orthopaedic doctor to review referrals to select which patients will be seen by a physiotherapist. A further knowledge gap relates to corticosteroid injection, a common non-surgical treatment for the most prevalent form of shoulder pain caused by subacromial pathologies (Diercks et al. 2014; Hambly et al. 2007; James et al. 2005; Jowett et al. 2013). Presently physiotherapists are only permitted to train in prescribing and injection in the UK (UK Government 2013), with similar models under investigation in some other countries (Health Workforce Australia). No studies have yet investigated prescribing and injection decisions of medical doctors compared with physiotherapists trained in these procedures. 
Therefore, we compared decisions made by a physiotherapist and a consultant orthopaedic surgeon in a representative sample of adult shoulder pain referred by general practitioners to hospital orthopaedics. Primary aims were to assess agreement regarding patient management, selection for subacromial corticosteroid injection and injection safety, and describe the reasoning underpinning decision discrepancies. Secondary aims were to describe the level of diagnostic and investigation agreement.

\section{METHODS}

\section{Design}

A blinded inter-rater decision-making reliability study, as described in the published RCT protocol (XXXX et al. 2014) investigating XXXX, was undertaken on XXXX between January 2013 and June 2014. Agreement between the physiotherapist and orthopaedic surgeon was an RCT entry and we have separated the inter-rater decision-making analysis from the RCT to enable it's full reporting. Other elements within this published protocol are reported separately. All participants provided written informed consent and were able to withdraw from the study at any point without impact upon their care.

\section{Ethical approval}

Ethical Approval was granted through the XXXX Human Research Ethics Committee, NHMRC code EC00160 (HREC/12/QGC/30; SSA/12/QGC/97), and XXXX Human Research Ethics Committee (MED/23/13/HREC). The trial was registered on the Australia and New Zealand Clinical Trials Registry XXXX 


\section{Participants}

Adults aged 18 years or over with a new orthopaedic shoulder referral from a general practitioner (GP) were recruited from the waiting list, if they were able to read and understand the trial literature and provide informed consent, and excluded if they knew the assessing physiotherapist or orthopaedic surgeon (eg, from previous consultation) or had not received a plain radiograph (X-Ray) of their affected shoulder in the previous 12 months (in keeping with local referral guidelines).

\section{Assessors}

The physiotherapist (XX) had 19 years musculoskeletal experience including qualifications in injection therapy and UK Non-Medical Prescribing. The orthopaedic surgeon (XX) was a staff specialist with 20 years of experience and a Fellow of the UK Royal College of Surgeons.

\section{Centres}

All participants were seen in orthopaedic outpatient settings, at XXXX.

\section{Intervention (clinical assessments)}

After the research assistant gained consent, all participants were independently examined the physiotherapist and the orthopaedic surgeon in variable order according to assessor availability. Patients were allocated 30 minutes with each assessor with the same information; the orthopaedic referral, the electronic hospital record, shoulder X-ray report, other results such as diagnostic imaging films and/or 
reports (MRI, CT, ultrasound scans) or pathology results that participants brought to the consultation. Each assessor performed a clinical assessment (history and physical examination) and completed a standardised assessment form for each participant, which was returned to the research assistant upon completion.

\section{Blinding}

Participants were blind to the profession of their assessors (i.e. they did not know which clinician was the physiotherapist and which was the surgeon), and were unaware of the clinical decisions of the assessors until all assessments had been completed. Assessors consulted with participants independently, in separate rooms and were blind to each other's findings.

\section{Outcome measures}

Participant characteristics collected by the research assistant prior to assessment included demographics, symptom duration, the Shoulder Pain and Disability Index (SPADI) which is a reliable and valid self-rating tool for people with shoulder pain (Angst et al. 2011; Roach et al. 1991); the worst shoulder pain severity over the past three days via a $100 \mathrm{~mm}$ visual analogue scale (VAS; $0=$ no pain, $100=$ worst pain imaginable); and the European Quality of Life five dimensions, five levels (EQ-5D-5L), converted to Australian values (Norman, Cronin, and Viney 2013). Assessors recorded their findings and clinical decisions on the paper assessment form. The research assistant was responsible for entry of data into the electronic database and coding of free text into categories for analysis. 
Primary outcomes investigated management and subacromial corticosteroid injection decisions made by each assessor. Management was recorded in two ways: dichotomous response (yes / no) to the question "is it appropriate to have initial non-surgical care?", and by free text "proposed management plan" responses. Subacromial corticosteroid injection decisions were investigated with dichotomous (yes / no) assessor responses to three questions focusing on different considerations within an injection decision: safety, "is it safe to offer injection today?"; whether there is symptomatic indication for the injection, "do clinical findings support the use of subacromial injection?"; and whether injection is the immediate treatment priority, "would you provide subacromial injection today?". Assessors also recorded reasons for not offering same-day injection via free text and under categories of safety, consent, other priority, need for prior investigation, or other.

Secondary outcomes were diagnoses and radiology or pathology investigations recorded in free text. Diagnoses were assigned to one of eight categories by the research assistant (if multiple contributory diagnoses were recorded, the first or primary diagnosis was used): 1. subacromial impingement syndrome (SAIS, SIS, bursitis), 2. glenohumeral osteoarthritis, 3. acromioclavicular osteoarthritis, 4. adhesive capsulitis, 5. rotator cuff tear (distinguished by it being listed above any other factors or requiring surgical repair), 6 . long head of biceps symptoms, 7. instability and / or labral pathology, and 8. pain from non-shoulder origin (including cervical referred, systemic inflammatory disorders, neurological or sensory disorders). 


\section{Sample size}

Sample size calculations for the randomised controlled trial within the published protocol (XXXX et al. 2014), assumed $\alpha=0.05$ and $\beta=0.2$, with a sample of 64 participants required for the RCT. We anticipated that $25 \%$ of all participants assessed, would enter the RCT. As all assessed participants would be eligible for the present agreement study, 256 were expected and 274 participants were ultimately recruited. This number exceeds the largest number of subjects $(n=100)$ previously reported by an inter-rater orthopaedic and physiotherapy agreement study (Razmjou et al. 2013). It also exceeds the number required to detect Kappa values over 0.6 with $90 \%$ power, assuming a null of Kappa $=0.4$ (Sim and Wright 2005), and the number required for agreement proportions of $30 \%$ to $100 \%$, with a relative error of $20 \%$ (Gwet 2010).

\section{Data analysis}

Data were analysed using SPSS software version 22 (IBM, Chicago USA), AgreeStat 2013.4, and AgreeStat 2015.5 for Excel (Advanced Analytics; Gaithersburg, MD, USA). Accuracy of the free text coding was assessed by two investigators ( $X X, X X)$, and at the completion of data entry the accuracy of the data entries ensured through comparison of the hard copy data and the data on the electronic database for 30 participants (every tenth participant), by one investigator (XX). Descriptive statistics were used to describe baseline characteristics. Four agreement coefficients were calculated for primary and secondary outcomes, to assess the level of agreement (inter-rater reliability) between the physiotherapist and the orthopaedic surgeon: Cohen's Kappa (Sim and Wright 2005), prevalence and bias adjusted Kappa 
(PABAK) (Sim and Wright 2005), Gwet's first order agreement coefficient (AC1)

(Gwet 2012), and percentage agreement. For free text "proposed management plan" responses, frequencies were calculated and presented descriptively.

Descriptive analysis of disagreements was also undertaken.

Kappa is commonly used for inter-rater agreement calculations (Gwet 2012; Sim and Wright 2005), yet its paradoxes are known to cause low values in the presence of high observed agreement (Cicchetti and Feinstein). To overcome this we used PABAK and Gwet's AC1 which both provide different alternative calculations for the agreement by chance (Gwet 2012). PABAK removes bias by bringing the expected agreement to an average value of 0.5 and should be interpreted alongside Cohen's Kappa (Sim and Wright 2005) and individual cell data (Chen et al. 2009). Gwet's AC1 removes the agreement by chance (Gwet 2012) and has been recommended for its stability in the presence of high inter-rater agreement (Wongpakaran et al. 2013). As previous shoulder inter-rater agreement studies used Kappa and PABAK (Razmjou et al. 2013), or Kappa with percentage agreement (Aiken and McColl 2008), we have presented the four measures, to both aid comparison with previous research and to demonstrate the consistency of our findings with the different measures. We applied the following established scale (Landis and Koch 1977) to the interpretation of the magnitude of Kappa and AC1 values: $<0.2$ poor, 0.21 - 0.4 fair, 0.41 - 0.6 moderate, $0.61-0.8$ substantial, $0.81-1$ near perfect agreement.

\section{RESULTS}


988 orthopaedic referrals were screened for eligibility by the research assistant according to the published protocol (XXXX et al. 2014). Of 305 attending between January 2013 and June 2014, 278 met the inclusion criteria. Three did not complete assessment at completion of the RCT protocol, and one participant with bilateral shoulder pain was excluded as the assessors each examined different shoulders. This left 274 participants who were examined by the physiotherapist and the orthopaedic surgeon, their characteristics are described in Table 1.

Table 1. Participant characteristics $(\mathbf{n}=\mathbf{2 7 4})$

\begin{tabular}{ll}
\hline Age years, mean (SD) & $57.9(13.0)$ \\
\hline Gender female \% & 49.0 \\
\hline Duration of shoulder symptoms months, mean (SD) & $21.7(36.5)$ \\
\hline Undertaking paid employment \% & 53.6 \\
\hline Total SPADI score /100, mean (SD) & $58.3(22.9)$ \\
\hline Worst pain last 3 days 100mm VAS, mean (SD) & $61.4(25.0)$ \\
\hline EQ-5D-5L health utility score, mean (SD) & $0.50(0.29)$ \\
\hline
\end{tabular}

\section{Primary Outcomes}

Management decisions:

There was near perfect agreement regarding initial management with surgery versus non-surgical care (Table 2), with the artificially low Cohen's Kappa (.28) a result of prevalence bias from high non-surgical agreement at 253 / 274, (Table 3). In the eight cases in which the surgeon said no to initial non-surgical care but the physiotherapist said yes, the physiotherapist also recommended orthopaedic review for surgery in four cases, and MRI in the other four. Conversely in nine cases the physiotherapist felt orthopaedic review was needed and said no to initial nonsurgical care but the surgeon said yes. In seven of these the surgeon opted for corticosteroid injection and recorded that surgery would be likely if response to 
injection was insufficient. In the remaining two cases the surgeon wanted to trial physiotherapy initially. Physiotherapy was the most frequent treatment recommendation by both assessors (223/ 274 cases). Overall, the physiotherapist recommended physiotherapy treatment in $86 \%$ of cases, and the surgeon in $91 \%$ of cases.

Table 2: Calculated agreement coefficients for the level of agreement between the physiotherapist and orthopaedic surgeon

\begin{tabular}{|c|c|c|c|c|c|c|c|}
\hline & \multicolumn{2}{|c|}{ Cohen's Kappa } & \multicolumn{2}{|c|}{ РАВAK } & \multicolumn{2}{|c|}{ AC1 } & \multirow[b]{2}{*}{$\begin{array}{c}\% \\
\text { agreement }\end{array}$} \\
\hline & $\begin{array}{l}\text { Value } \\
\text { (SEM) }\end{array}$ & $95 \% \mathrm{Cl}$ & $\begin{array}{l}\text { Value } \\
\text { (SEM) }\end{array}$ & $95 \% \mathrm{Cl}$ & $\begin{array}{l}\text { Value } \\
\text { (SEM) }\end{array}$ & $95 \% \mathrm{Cl}$ & \\
\hline Initial management ${ }^{*}$ & $.28(0.1)$ & $.04-.53$ & $.87(.03)$ & $.81-.93$ & $.93(.02)$ & $.90-.93$ & 94 \\
\hline Safety of injection" & $.65(.06)$ & $.53-.76$ & $.78(.04)$ & $.71-.86$ & $.85(.02)$ & $.79-.91$ & 89 \\
\hline Subacromial pain $\$$ & $.43(.06)$ & $.31-.56$ & $.64(.04)$ & $.55-.73$ & $.74(.03)$ & $.66-.81$ & 82 \\
\hline Same-day inject^^ & $.42(.05)$ & $.32-.53$ & $.45(.05)$ & $.39-.55$ & $.48(.05)$ & $.33-.53$ & 72 \\
\hline Investigations ${ }^{\&}$ & $.52(.07)$ & $.38-.66$ & $.85(.02)$ & $.80-.90$ & $.87(.02)$ & $.83-.91$ & 88 \\
\hline Diagnosis $^{+}$ & $.53(.05)$ & $.44-.62$ & $.70(.03)$ & $.64-.76$ & $.72(.03)$ & $.66-.78$ & 74 \\
\hline $\begin{array}{l}\text { *Initial management } \\
\# \$ \wedge \text { Categories: Yes / } \\
\text { \& Nil, XRay, MRI, CT sC } \\
{ }^{+} \text {Diagnostic categorie } \\
\text { osteoarthritis, 3. Acr } \\
\text { 6. Long head biceps } \\
\text { origin. } \\
\text { Subacromial pain = cli } \\
\text { SEM = standard error } \\
\text { adjusted Kappa, AC1 }\end{array}$ & $\begin{array}{l}\text { surgical / } n \\
\text { lo } \\
\text { an, blood } t \\
\text { s: } 1 \text {. Subac } \\
\text { omioclavic } \\
\text { ymptoms, } \\
\text { nical findin } \\
\text { of the mea } \\
\text { Gwet's fir }\end{array}$ & $\begin{array}{l}\text { ests } \\
\text { omial imr } \\
\text { Alar osteo } \\
\text { 7. Instabil } \\
\text { gs suppor } \\
\text { n; } \mathrm{Cl}=\text { cor } \\
\text { st order a }\end{array}$ & $\begin{array}{l}\text { ingement } \\
\text { arthritis, } 4 . \\
\text { ity / labral } \\
\text { the use o } \\
\text { fidence in } \\
\text { greement }\end{array}$ & $\begin{array}{l}\text { syndrome } \\
\text { Adhesive } \\
\text { patholog } \\
\text { f subacror } \\
\text { terval, PA } \\
\text { coefficien }\end{array}$ & $\begin{array}{l}\text { 2. Glenoh } \\
\text { capsulitis, } \\
\text { 8.Pain fro } \\
\text { hial injectic } \\
\text { BAK = prev }\end{array}$ & $\begin{array}{l}\text { umeral } \\
\text { 5. Rotator } \\
\text { m non-sh } \\
\text { alence an }\end{array}$ & $\begin{array}{l}\text { cuff tear, } \\
\text { oulder }\end{array}$ \\
\hline
\end{tabular}

\begin{tabular}{llll}
\hline \multicolumn{4}{l}{ Table 3: Management: “is it appropriate to have initial non-surgical care?” } \\
\hline \multicolumn{4}{c}{ Orthopaedic Surgeon } \\
\hline
\end{tabular}


Injection decisions:

There was substantial to near perfect agreement regarding whether it was safe to inject (Table 2). Of the 36 cases in which the surgeon felt it was unsafe to inject on the day of assessment, the physiotherapist reached the same conclusion in 35 (Table 4). In the one remaining case, the patient reported being well but test results from recent endoscopy were not available on the day of assessment. The physiotherapist rated this as a precaution rather than a contraindication to injection. Conversely in 28 cases the physiotherapist perceived a safety barrier yet the surgeon felt it would be safe to inject. The physiotherapist's reasons were; the possibility of a concurrent infection (such as skin, dental, urinary or other) needing further medical review before injection ( $N=20)$, possible need for prophylactic antibiotics prior to injection due to a prosthetic heart valve $(\mathrm{N}=3)$, history of possible fracture $(\mathrm{N}=2)$, and poor diabetic control $(\mathrm{N}=1)$.

Table 4: Injection decisions

\begin{tabular}{|c|c|c|c|c|}
\hline \multirow{10}{*}{$\begin{array}{l}\frac{n}{0} \\
\frac{0}{0} \\
\frac{0}{0} \\
\frac{c}{\Delta} \\
\frac{0}{5} \\
\frac{1}{a}\end{array}$} & \multicolumn{4}{|c|}{ Orthopaedic Surgeon } \\
\hline & & Yes & No & Total \\
\hline & Yes & 210 & 1 & 211 \\
\hline & No & 28 & 35 & 63 \\
\hline & Total & 238 & 36 & 274 \\
\hline & \multicolumn{4}{|c|}{ Subacromial symptoms present? } \\
\hline & & Yes & No & Total \\
\hline & Yes & 197 & 42 & 239 \\
\hline & No & 7 & 28 & 35 \\
\hline & Total & 204 & 70 & 274 \\
\hline
\end{tabular}


Would you provide subacromial injection today?

\begin{tabular}{cccc}
\hline & Yes & No & Total \\
Yes & 66 & 14 & $\mathbf{8 0}$ \\
No & 62 & 132 & $\mathbf{1 9 4}$ \\
Total & $\mathbf{1 2 8}$ & $\mathbf{1 4 6}$ & $\mathbf{2 7 4}$ \\
\hline
\end{tabular}

There were substantial levels of agreement on the presence or absence of symptoms to support the use of subacromial injection (Table 2). Again prevalence bias lowers Cohen's Kappa to .43 because of a high incidence of agreement between assessors (table 4) on the presence of subacromial symptoms (197/274 cases).

Table 2 reveals moderate agreement regarding same-day delivery of subacromial corticosteroid injection. In most cases assessors agreed to provide, or not provide, same day injection (Table 4). There were 14 cases of disagreement, in which the physiotherapist said yes and surgeon said no. The surgeon's reasons were: eight cases of dual pathology in which the surgeon chose to inject another structure over the subacromial space as first priority (long head of biceps sheath in six cases, acromioclavicular joint in two cases); in four cases the surgeon referred for initial physiotherapy; and two cases the surgeon requested MRI in the first instance. Conversely there were 62 cases in which the surgeon opted to provide same-day subacromial injection but the physiotherapist did not. A detailed breakdown of the reasons is contained in Table 5.

Table 5: Physiotherapist reasons for not choosing same-day subacromial injection, when the surgeon did chose to deliver same day subacromial injection.

Physiotherapist decision No. Reason provided by physiotherapist (number of cases) 


\begin{tabular}{lcl}
\hline $\begin{array}{l}\text { Physiotherapy treatment } \\
\text { is higher priority }\end{array}$ & 17 & $\begin{array}{l}\text { Spine +/- shoulder physiotherapy treatment (10) } \\
\text { Shoulder physiotherapy treatment (7) }\end{array}$ \\
\hline $\begin{array}{l}\text { Safety concerns prevent } \\
\text { injection on the day }\end{array}$ & 15 & $\begin{array}{l}\text { Symptoms of unrelated infection for medical review (12) } \\
\text { Patient unable to supply names of a drug being used (1) } \\
\text { Prophylactic antibiotic consideration-prosthetic cardiac valve (1) } \\
\text { Allergic skin reaction to another agent already present (1) }\end{array}$ \\
\hline $\begin{array}{l}\text { Orthopaedic review } \\
\text { needed prior to an } \\
\text { injection }\end{array}$ & 13 & $\begin{array}{l}\text { Cuff tear for MRI +/- surgical consideration (7) } \\
\text { MRI shoulder due to diagnostic uncertainty (4) } \\
\text { MRI cervical spine (1) } \\
\text { Stable shoulder fracture present, for orthopaedic opinion (1) }\end{array}$ \\
\hline $\begin{array}{l}\text { Presentation not likely to } \\
\text { respond to subacromial } \\
\text { injection }\end{array}$ & 5 & $\begin{array}{l}\text { Significant rotator cuff tear (2) } \\
\text { Long head of biceps pain source (1) } \\
\text { Osteoarthritis too significant (1) } \\
\text { Cervical spine cause (1) }\end{array}$ \\
\hline $\begin{array}{l}\text { Other injection is higher } \\
\text { priority }\end{array}$ & 5 & $\begin{array}{l}\text { Long head of biceps sheath (5) } \\
\text { Consent not gained }\end{array}$ \\
\hline $\begin{array}{l}\text { Possible inflammatory } \\
\text { pathology }\end{array}$ & 3 & Patient declined injection when offered by physiotherapist (4) \\
\hline
\end{tabular}

\section{Secondary Outcomes}

Investigation requests revealed near perfect agreement $(\mathrm{AC} 1=.87)$ by type (Table 2$)$, and similar volume (Table 6). In 220 cases both assessors agreed that no further investigations were required. Overall the physiotherapist recommended no further investigation in 226 cases, the surgeon in 245 . The physiotherapist recommended MRI in $42 / 274$ (15\%) of cases, the surgeon in $28 / 274$ (10\%). In 22 out of the 28 cases both requested MRI, and in four of these cases both assessors requested an MRI and CT scan simultaneously for arthroplasty planning. The physiotherapist recommended MRI in another 20 other cases when the surgeon didn't, however in half of these cases the surgeon's plan was to review in six weeks and progress to MRI or surgery if initial management had not been successful. 


\begin{tabular}{|c|c|c|c|c|c|c|c|}
\hline \multirow{6}{*}{ 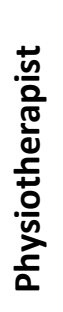 } & & Nil & XRay & MRI & CT Scan & Bloods & Total \\
\hline & Nil & 220 & 1 & 5 & 0 & 0 & 226 \\
\hline & XRay & 2 & 0 & 1 & 0 & 0 & 3 \\
\hline & MRI & 20 & 0 & 22 & 0 & 0 & 42 \\
\hline & CT & 1 & 0 & 0 & 4 & 0 & 5 \\
\hline & Bloods & 2 & 0 & 0 & 0 & 0 & 2 \\
\hline & Total & 245 & 1 & 28 & 4 & 0 & \\
\hline
\end{tabular}

All participants attended with an XRay report of the painful shoulder less than 12 months old.

Diagnosis:

Overall, there was substantial agreement $(A C 1=0.72)$ between the two assessors on categorised primary shoulder pain diagnoses (Table 2). The frequencies with which each assessor recorded primary diagnoses in each of the eight diagnostic categories, and the agreement between assessors are detailed in Table 7. Subacromial impingement was the most common primary diagnosis, made by the orthopaedic surgeon in 188 cases, the physiotherapist in 170 cases, with agreement in 148 cases. Other primary diagnoses were recorded less frequently, with varying levels of agreement as detailed in Table 7.

Table 7: Diagnosis made by each assessor

\begin{tabular}{cccccccccc}
\hline \multicolumn{1}{c}{ Orthopaedic Surgeon } \\
\hline Category & $\mathbf{1}$ & $\mathbf{2}$ & $\mathbf{3}$ & $\mathbf{4}$ & $\mathbf{5}$ & $\mathbf{6}$ & $\mathbf{7}$ & $\mathbf{8}$ & Total \\
\hline $\mathbf{1}$ & 148 & 1 & 3 & 3 & 6 & 6 & 1 & 2 & 170 \\
\hline $\mathbf{2}$ & 2 & 8 & 0 & 0 & 0 & 0 & 0 & 0 & 10 \\
\hline $\mathbf{3}$ & 4 & 1 & 7 & 0 & 0 & 1 & 0 & 0 & 13 \\
\hline $\mathbf{4}$ & 4 & 0 & 0 & 12 & 0 & 0 & 0 & 0 & 16 \\
\hline $\mathbf{5}$ & 6 & 0 & 0 & 0 & 7 & 0 & 0 & 0 & 13 \\
\hline $\mathbf{0}$ & 9 & 1 & 0 & 0 & 1 & 5 & 0 & 0 & 16 \\
\hline $\mathbf{0}$ & 3 & 0 & 0 & 0 & 0 & 0 & 2 & 0 & 5 \\
\hline $\mathbf{8}$ & 12 & 0 & 3 & 0 & 0 & 1 & 1 & 13 & 30 \\
\hline Total & 188 & 11 & 13 & 15 & 14 & 13 & 4 & 15 & 273 \\
\hline $\begin{array}{l}\text { Categories 1. Subacromial impingement syndrome, 2. Glenohumeral } \\
\text { osteoarthritis, 3. Acromioclavicular osteoarthritis, 4. Adhesive capsulitis, }\end{array}$
\end{tabular}


5. Rotator cuff tear, 6. Long head biceps symptoms, 7. Instability / labral pathology, 8.Pain of non-shoulder origin.

\section{DISCUSSION}

Results demonstrate that the physiotherapist makes safe and very similar decisions to the orthopaedic surgeon in relation to care delivery, prescription of corticosteroid injection, investigation and diagnosis. These findings provide preliminary support for agreement between these two professions and have relevance to models of care which complement or substitute medical doctors with physiotherapists to improve access for patients, service productivity and workforce shortages (Brooks, Robinson, and Ellis 2008; Comans et al. 2014; Duckett 2005). In previous studies orthopaedic doctors first screened referrals to exclude more complex patients and/or those thought more likely to need surgery (Aiken and McColl 2008; Bornhöft, Larsson, and Thorn 2015; Daker-White et al. 1999; Ludvigsson et al. 2011; MacKay et al. 2009; Samsson and Larsson 2014; Weale and Bannister 1995). We did not exclude more complex referrals and therefore our results indicate that an experienced physiotherapist can safely and appropriately manage the breadth of GP orthopaedic shoulder referrals, suggesting that screening of referrals by orthopaedic doctors prior to physiotherapist assessment may be unnecessary.

Previously physiotherapists have more frequently recommended education and physiotherapy exercises than orthopaedic doctors (Aiken et al. 2008; Daker-White et al. 1999; Desmeules et al. 2013), yet we found near perfect agreement and minimal overall difference in the rate of referral to physiotherapy. Our findings of lower 
injection recommendation rates by the physiotherapist contrast with two previous studies (Desmeules et al. 2013; Razmjou et al. 2013). However, ours is the first study to investigate this with a physiotherapist qualified to inject and prescribe.

Agreement was near perfect regarding the safety aspects of injection and substantial regarding the presence of subacromial symptoms. Whilst still moderate, agreement was less regarding whether to deliver an immediate injection, with $14 / 274$ cases in which the physiotherapist chose to inject but the surgeon did not, and 62/274 cases in which the surgeon chose to immediately inject but the physiotherapist did not; few of which were due to disagreement about whether the presentation may be amenable to subacromial injection, but rather reflected different prioritisation of non-surgical treatment options, with the physiotherapist less inclined than the surgeon to start with corticosteroid injection (Table 5). We consider this more conservative approach to injection by the physiotherapist to be appropriate, and further research could establish whether this finding is a consistent feature of physiotherapy prescribing / injection practice.

This study has some limitations. Generalizability is limited with just one orthopaedic surgeon and one physiotherapist. While this is consistent with previously published shoulder decision-making comparisons (Aiken and McColl 2008; Razmjou et al. 2013), it means that specific findings may have limited generalizability beyond this study. The use of only one physiotherapist was due to the study protocol (XXXX et al. 2014) including injection by a physiotherapist (reported separately), and as this is not normally permitted in Australia, additional qualified physiotherapists were not available. Future research with more professionals could verify our findings. 
Furthermore, intra-professional decision-making has not previously been reported, and this could provide a more robust basis for inter-professional comparison. A second limitation was the exclusion of participants without a reported X-Ray less than 12 months old. This met departmental referral requirements and helped prevent clinic delays but also reduced the need for assessors to interpret unreported imaging. Thirdly, we were unable to formally randomize the order of the participant examination (physiotherapist or surgeon first) due to the practicalities of room availability in an outpatient clinic setting. Instead, participants were pseudorandomized according to which health practitioner was first available at the time of their appointment. Whilst appointments were allocated on a 30-minute basis, we did not record actual consultation times. A final limitation is that the collection of followup clinical outcomes was beyond the scope of the present study. This data could provide further insight, particularly in cases of assessor disagreement.

In conclusion, this study compared one experienced physiotherapist qualified in prescribing and corticosteroid injection, with one orthopaedic surgeon and found near perfect agreement between the two regarding clinical decision making (investigation, surgical or non-surgical management and subacromial corticosteroid injection safety) for shoulder patients referred to an Australian public hospital orthopaedic department. There were differences in the priority given to different non-surgical treatment options, with a more conservative approach by the physiotherapist regarding subacromial corticosteroid injection as the first and immediate treatment. This study suggests that screening of referrals by orthopaedic doctors prior to assessment by a suitably qualified physiotherapist may be 
unnecessary, and found no clinical, safety or cost basis for legislative or funding

policy impediments to appropriately trained physiotherapists selecting and referring

shoulder pain patients for subacromial corticosteroid injection.

\section{REFERENCES}

Aiken, A. B., M. M. Harrison, M. Atkinson, and J. Hope. 2008. "Easing the burden for joint replacement wait times: the role of the expanded practice physiotherapist." Healthc $Q$ 11(2): 62-6.

Aiken, A. B. and M. A. McColl. 2008. "Diagnostic and treatment concordance between a physiotherapist and an orthopedic surgeon--a pilot study." $J$ Interprof Care 22(3): 253-61.

Angst, F., H.-K. Schwyzer, A. Aeschlimann, B. R. Simmen, and J. Goldhahn. 2011. "Patient outcomes in rheumatology, 2011: a review of measures. Measures of adult shoulder function." Arthritis Care \& Research (2151464X) 63(11): S17488.

Australian Government Productivity Commission. 2011. "Caring for Older Australians, Report No. 53, Final Inquiry Report." Canberra.

Bornhöft, L., M. E. H. Larsson, and J. Thorn. 2015. "Physiotherapy in Primary Care Triage - the effects on utilization of medical services at primary health care clinics by patients and sub-groups of patients with musculoskeletal disorders: a case-control study." Physiotherapy theory and practice 31(1): 4552.

Brooks, P. M., L. Robinson, and N. Ellis. 2008. “Options for expanding the health workforce." Australian Health Review : A Publication of the Australian Hospital Association 32(1): 156.

Buchbinder, R., M. P. Staples, E. M. Shanahan, and J. F. Roos. 2013. “General practitioner management of shoulder pain in comparison with rheumatologist expectation of care and best evidence: an Australian national survey." Plos One 8(4): e61243-e43.

Chen, G., P. Faris, B. Hemmelgarn, R. L. Walker, and H. Quan. 2009. “Measuring agreement of administrative data with chart data using prevalence unadjusted and adjusted kappa." BMC Medical Research Methodology 9(1): 5-5.

Cicchetti, D. V. and A. R. Feinstein. "High agreement but low kappa: II. Resolving the paradoxes." Journal Of Clinical Epidemiology 43(6): 551-58.

Comans, T., M. Raymer, S. O'Leary, D. Smith, and P. Scuffham. 2014. "Costeffectiveness of a physiotherapist-led service for orthopaedic outpatients." Journal of Health Services Research \& Policy 19(4): 216-23.

Daker-White, G., A. J. Carr, I. Harvey, G. Woolhead, G. Bannister, I. Nelson, and M. Kammerling. 1999. "A randomised controlled trial. Shifting boundaries of doctors and physiotherapists in orthopaedic outpatient departments." Journal Of Epidemiology And Community Health 53(10): 643-50.

Department of Health. 2006. "The musculoskeletal services framework: a joint responsibility." London. 
Desmeules, F., J.-S. Roy, J. C. MacDermid, F. Champagne, O. Hinse, and L. J. Woodhouse. 2012. "Advanced practice physiotherapy in patients with musculoskeletal disorders: a systematic review." BMC Musculoskeletal Disorders 13: 107-07.

Desmeules, F., P. Toliopoulos, J.-S. Roy, L. J. Woodhouse, M. Lacelle, M. Leroux, S. Girard, D. E. Feldman, and J. C. Fernandes. 2013. "Validation of an advanced practice physiotherapy model of care in an orthopaedic outpatient clinic." BMC Musculoskeletal Disorders 14: 162-62.

Diercks, R., C. Bron, O. Dorrestijn, C. Meskers, R. Naber, T. de Ruiter, J. Willems, J. Winters, H. J. van der Woude, and A. Dutch Orthopaedic. 2014. "Guideline for diagnosis and treatment of subacromial pain syndrome: a multidisciplinary review by the Dutch Orthopaedic Association." Acta orthopaedica 85(3): 31422.

Dorrestijn, O., K. Greving, W. J. van der Veen, K. van der Meer, R. L. Diercks, J. C. Winters, and M. Stevens. 2011. "Patients with shoulder complaints in general practice: consumption of medical care." Rheumatology (Oxford, England) 50(2): 389-95.

Duckett, S. J. 2005. "Health workforce design for the 21st century." Australian Health Review 29(2): 201-10.

Gwet, K. 2010. "Inter-Rater Reliability Discussion Corner" [accessed on August 15, 2010]. Available at: http://agreestat.com/blog irr/sample size determination.html.

Gwet, K. 2012. Handbook of inter-rater reliabiilty. They definitive guide to measuring the extent of agreement between raters. Gaithersurg, MD: Advanced Analytics

Hambly, N., P. Fitzpatrick, P. MacMahon, and S. Eustace. 2007. "Rotator Cuff Impingement: Correlation Between Findings on MRI and Outcome After Fluoroscopically Guided Subacromial Bursography and Steroid Injection." American Journal of Roentgenology 189(5): 1179-84.

Health Workforce Australia. "Health Professionals Prescribing Pathway (HPPP) Project -Final Report."

James, M., E. A. Stokes, E. Thomas, K. Dziedzic, and E. M. Hay. 2005. "A cost consequences analysis of local corticosteroid injection and physiotherapy for the treatment of new episodes of unilateral shoulder pain in primary care." Rheumatology (Oxford, England) 44(11): 1447-51.

Jowett, S., D. P. Crawshaw, P. S. Helliwell, E. M. A. Hensor, E. M. Hay, and P. G. Conaghan. 2013. "Cost-effectiveness of exercise therapy after corticosteroid injection for moderate to severe shoulder pain due to subacromial impingement syndrome: a trial-based analysis." Rheumatology (Oxford, England) 52(8): 1485-91.

Landis, J. R. and G. G. Koch. 1977. "The Measurement of Observer Agreement for Categorical Data." Biometrics 33(1): 159-74.

Linsell, L., J. Dawson, K. Zondervan, P. Rose, T. Randall, R. Fitzpatrick, and A. Carr. 2006. "Prevalence and incidence of adults consulting for shoulder conditions in UK primary care; patterns of diagnosis and referral." Rheumatology (Oxford, England) 45(2): 215-21.

Ludvigsson, M. L., P. Enthoven, u. Linköpings, h. Institutionen för medicin och, s. Avdelningen för, Sjukgymnastik, and Hälsouniversitetet. 2011. "Evaluation of 
physiotherapists as primary assessors of patients with musculoskeletal disorders seeking primary health care." Physiotherapy 98(2): 131.

MacKay, C., A. M. Davis, N. Mahomed, and E. M. Badley. 2009. "Expanding roles in orthopaedic care: a comparison of physiotherapist and orthopaedic surgeon recommendations for triage." Journal of evaluation in clinical practice 15(1): 178-83.

XXXX 15(1): 503.

Murray, C. et al. 2012. "Disability-adjusted life years (DALYs) for 291 diseases and injuries in 21 regions, 1990-2010: a systematic analysis for the Global Burden of Disease Study 2010." Lancet 380(9859): 2197.

Norman, R., P. Cronin, and R. Viney. 2013. "A Pilot Discrete Choice Experiment to Explore Preferences for EQ-5D-5L Health States." Applied Health Economics and Health Policy 11(3): 287-98.

Parsons, S., A. Breen, N. E. Foster, L. Letley, T. Pincus, S. Vogel, and M. Underwood. 2007. "Prevalence and comparative troublesomeness by age of musculoskeletal pain in different body locations." Family practice 24(4): 30816.

Picavet, H. S. J. and J. S. A. G. Schouten. 2003. "Musculoskeletal pain in the Netherlands: prevalences, consequences and risk groups, the DMC3-study." Pain 102(1-2): 167-78.

Queensland Health. 2013. "N/OPSC \& MDS PSC3 database." Queensland Health Electronic Publishing Service, Patient Safety Unit, Brisbane.

Razmjou, H., S. Robarts, D. Kennedy, C. McKnight, A. M. MacLeod, and R. Holtby. 2013. "Evaluation of an Advanced-Practice Physical Therapist in a Specialty Shoulder Clinic: Diagnostic Agreement and Effect on Wait Times." Physiotherapy Canada 65(1): 46-55.

Roach, K. E., E. Budiman-Mak, N. Songsiridej, and Y. Lertratanakul. 1991. "Development of a shoulder pain and disability index." Arthritis Care And Research: The Official Journal of The Arthritis Health Professions Association 4(4): 143-49.

Samsson, K. and M. E. H. Larsson. 2014. "Physiotherapy screening of patients referred for orthopaedic consultation in primary healthcare - a randomised controlled trial." Manual therapy 19(5): 386.

Sim, J. and C. C. Wright. 2005. "The Kappa Statistic in Reliability Studies: Use, Interpretation, and Sample Size Requirements." Physical Therapy 85(3): 257.

Stanhope, J., K. Grimmer-Somers, S. Milanese, S. Kumar, and J. Morris. 2012. "Extended scope physiotherapy roles for orthopedic outpatients: an update systematic review of the literature." Journal Of Multidisciplinary Healthcare 5: 37-45.

Taylor, W. 2005. "Musculoskeletal pain in the adult New Zealand population: prevalence and impact." The New Zealand medical journal 118(1221): U1629.

The Kings Fund. 2015. "How much money does the NHS need?" [accessed on 1.4.2015, 2015]. Available at:

http://www.kingsfund.org.uk/projects/verdict/how-much-money-does-nhsneed.

UK Government. 2013. "Millions of patients to benefit from easier access to medication and fewer trips to hospitals." D. o. Health.

Urwin, M., D. Symmons, T. Allison, T. Brammah, H. Busby, M. Roxby, A. Simmons, and G. Williams. 1998. "Estimating the burden of musculoskeletal 
disorders in the community: the comparative prevalence of symptoms at different anatomical sites, and the relation to social deprivation." Annals of The Rheumatic Diseases 57(11): 649-55.

Vos, T.A. D. et al. 2012. "Years lived with disability (YLDs) for 1160 sequelae of 289 diseases and injuries 1990-2010: a systematic analysis for the Global Burden of Disease Study 2010." Lancet 380(9859): 2163-96.

Weale, A. E. and G. C. Bannister. 1995. "Who should see orthopaedic outpatients--physiotherapists or surgeons?" Annals Of The Royal College of Surgeons Of England 77(2 Suppl): 71-73.

Wongpakaran, N., T. Wongpakaran, D. Wedding, and K. L. Gwet. 2013. “A comparison of Cohen's Kappa and Gwet's AC1 when calculating inter-rater reliability coefficients: a study conducted with personality disorder samples." BMC Medical Research Methodology 13(1): 61-61. 